\title{
Coil Navigation - Imaging for Juvenile nasopharyngeal Angiofibroma
}

\author{
PARESH NAIK ${ }^{1}$, Emma Richards ${ }^{1}$, and Shahz Ahmed ${ }^{2}$ \\ ${ }^{1}$ Queen Elizabeth Hospital Birmingham \\ ${ }^{2}$ Queen Elizabeth Hospital
}

August 4, 2020

\begin{abstract}
Abstract Juvenile nasal angiofibroma is a benign tumour mainly found in adolescent males. Its primary treatment is surgical excision. Preoperative embolisation is widely peformed prior to the surgery. We propose some changes to the approach to the pre-operative navigation imaging that may help improve the resection.
\end{abstract}

Letter to the Editor

Coil Navigation - Imaging for Juvenile nasopharyngeal Angiofibroma

Abstract

Juvenile nasal angiofibroma is a benign tumour mainly found in adolescent males. Its primary treatment is surgical excision. Preoperative embolisation is widely peformed prior to the surgery. We propose some changes to the approach to the pre-operative navigation imaging that may help improve the resection.

Dear Editor,

Juvenile nasopharyngeal angiofibroma (JNA) is a locally aggressive tumour which accounts for about $0.05 \%$ of all head and neck tumours. It is the most common tumour presenting in the nose and nasopharynx and is almost exclusively seen in young adolesent men. The most common site of JNA is the sphenopalatine foramen and it extends from here into the pterygopalatine fossa, infratemporal fossa, nasal cavity , paranasal sinuses, cavernous sinus, intracranial cavity, infraorbital fissure and orbit, paraphayrngeal space and neck. Routine preoperative imaging includes computed tomography (CT) and magnetic resonance imaging (MRI), which help in confirming the diagnosis and extent of the disease. CT, MRI, and angiography assist in defining the location, the relationship to important neurovascular structures, and assessment of the blood supply which facilitate surgical planning.(1)

Since the evolution of surgical navigation technology, the ease of surgical resection has greatly improved. CT is considered superior in outlining bony landmarks, invasion to neighbouring structures and demonstrating any bony erosion. Bilateral Carotid angiography is essential to recognise the blood supply of the JNA. Pre-operative embolisation is widely performed prior to surgery, however some surgeons hold the view that embolisation can give the impression of shrinkage of the tumour, leading to incomplete resection $(2,3)$.

We propose some changes to the approach to pre-operative navigation imaging that may help improve resection rates. For the past nine years we have been undertkaing navigation protocol CT imaging immeditately after embolisation (Figure).

The overall benefit of these post embolisation scans is the accurate identification of the extent of the tumour post embolisation, highlighting important landmarks to the operating surgeon, allowing the surgeon to use 
intraoperative surgical navigation to reach the embolisaiton coils and the main feeding arteries to the tumour and ultimatley achieving more radical resections.

\section{References :}

1.López, F., Triantafyllou, A., Snyderman, C. H., Hunt, J. L., Suárez, C., Lund, V. J., ... Ferlito, A. (2017). Nasal juvenile angiofibroma: Current perspectives with emphasis on management. Head $\& 3$ Neck, 39(5), 1033-1045. doi:10.1002/hed.24696

2.Lloyd G, Howard D, Lund VJ, Savy L. Imaging for juvenile angiofibroma. J Laryngol Otol 2000;114:727730 .

3.Mann WJ, Jecker P, Amedee RG. Juvenile angiofibromas: changing surgical concept over the last 20 years. Laryngoscope 2004;114:291-293
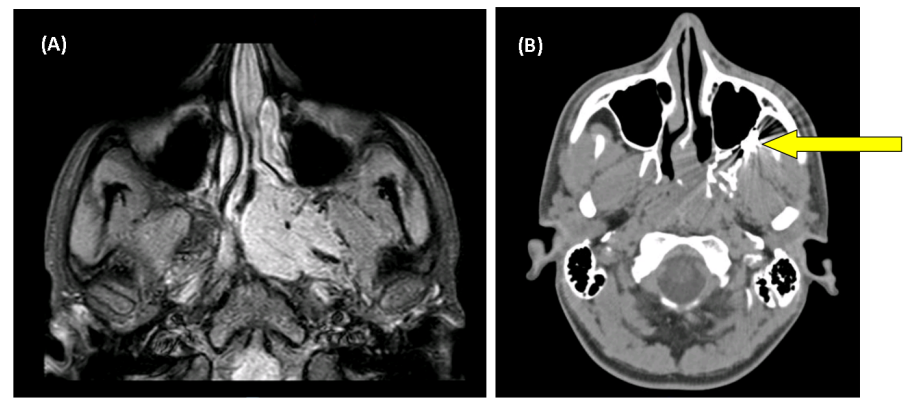